\title{
Review \\ Bench-to-bedside review: Bacterial virulence and subversion of host defences
}

\author{
Steven AR Webb ${ }^{1}$ and Charlene M Kahler²
}

\begin{abstract}
${ }^{1}$ School of Medicine and Pharmacology and School of Population Health, University of Western Australia, Intensive Care Unit, Royal Perth Hospital, Wellington Street, Perth, Western Australia 6000, Australia

${ }^{2}$ School of Biomolecular, Biochemical and Chemical Science, Microbiology M502, University of Western Australia, 35 Stirling Highway, Crawley, Western Australia 6909, Australia
\end{abstract}

Corresponding author: Steven AR Webb, steve.webb@uwa.edu.au

Published: 10 November 2008

This article is online at http://ccforum.com/content/12/6/234

Critical Care 2008, 12:234 (doi:10.1186/cc7091)

(c) 2008 BioMed Central Ltd

\begin{abstract}
Bacterial pathogens possess an array of specific mechanisms that confer virulence and the capacity to avoid host defence mechanisms. Mechanisms of virulence are often mediated by the subversion of normal aspects of host biology. In this way the pathogen modifies host function so as to promote the pathogen's survival or proliferation. Such subversion is often mediated by the specific interaction of bacterial effector molecules with host encoded proteins and other molecules. The importance of these mechanisms for bacterial pathogens that cause infections leading to severe community-acquired infections is well established. In contrast, the importance of specialised mechanisms of virulence in the genesis of nosocomial bacterial infections, which occur in the context of local or systemic defects in host immune defences, is less well established. Specific mechanisms of bacterial resistance to host immunity might represent targets for therapeutic intervention. The clinical utility of such an approach for either prevention or treatment of bacterial infection, however, has not been determined.
\end{abstract}

\section{Introduction}

The interaction of pathogenic bacteria with the host plays a central role in many forms of critical illness. As well as being a common trigger of sepsis that necessitates admission to the intensive care unit (ICU), bacterial infections are responsible for the majority of nosocomial infections that occur in these patients.

For over 60 years the mainstay of treatment of bacterial infection has been antibiotics. There is overwhelming evidence, albeit derived from observational studies, that administration of antibiotics improves survival of patients with severe sepsis [1]. Antibiotic treatment, however, is often not sufficient to improve mortality [2]. Although the prophylactic use of antibiotics can reduce nosocomial infection, the practice remains controversial and it cannot eliminate nosocomial infection [3]. Of substantial concern is the increasing problem of antibiotic resistance - a problem that ICUs both contribute to as well as suffer from [4]. Despite the rising incidence of antibiotic resistance in many bacterial pathogens, interest in antibiotic drug discovery by commercial entities is in decline [5].

Bacterial virulence is 'the ability to enter into, replicate within, and persist at host sites that are inaccessible to commensal species' [6]. As a consequence of the availability of whole genome sequencing and high-throughput techniques for the identification of virulence genes from many bacterial pathogens, the past 10 to 15 years have witnessed a revolution in the understanding of bacterial virulence. While virulence factors such as capsules and serum resistance have been known for decades, and are often necessary if not sufficient for infection, a much wider array of more specialised determinants of virulence has now been characterised. Many of these mechanisms of virulence are now defined at precise molecular and genetic levels; however, the ultimate clinical relevance of this knowledge remains uncertain. With the possible exception of lincosamides, such as clindamycin, all existing antibiotics target bacterial products that are essential for survival of the organism, leading to bacterial death, and do not target mechanisms of virulence. Whether virulence will ever be a useful and drugable target remains speculative but, in the presence of increasing antibiotic resistance and decreasing antibiotic drug development, it is a potentially important question.

\section{Principles of bacterial virulence}

Although encounters between bacteria and humans occur continuously, the establishment of infection after such contact is extremely rare. The ability of the human body to prevent most interactions with bacteria resulting in harm is a testament to the multilayered defences that prevent the

$\mathrm{ICU}=$ intensive care unit; MAC = membrane attack complex; T3SS $=$ type III secretion system. 
establishment of bacterial infection. The most effective of these defences are the barrier function of epithelial surfaces and innate immune responses - both of which are deeply evolutionarily conserved [7].

Just as humans possess sophisticated and effective defences against infection, the bacteria that are capable of infection possess equally sophisticated mechanisms to counteract and overcome the human defences allayed against them. The core competencies of a potentially pathogenic bacterium are to gain access to the body; to attain a unique niche; to avoid, subvert or circumvent innate host defences; to evade acquired specific immune responses; to acquire necessary nutrients; to multiply or persist; to cause tissue damage or disease; and to exit and transmit infection to new hosts [8]. Pathogenic bacteria possess specific mechanisms to achieve each of these aims, and it is the possession of these mechanisms that distinguishes pathogenic bacteria from nonpathogens. These mechanisms of virulence are genetically encoded by so-called virulence genes, and possession of such genes distinguishes pathogenic bacteria from nonpathogens. There is a spectrum of pathogenic potential among pathogenic bacteria - from those that are opportunistic pathogens, only capable of virulent behaviour in the presence of local or systemic defects of host defences, through to pathogens, which might be termed professional, capable of pathogenic behaviour in the presence of normally functioning host defences. Within broad limits the latter are much more responsible for severe infection that necessitates ICU admission, with clear attributable mortality. In contrast, the virulence and harm caused to the host by bacteria that cause nosocomial infection is an open question.

There is little work that compares virulence - for example, by evaluation of the lethal dose in animal models - of nosocomial versus community-acquired pathogens. The presence of invasive devices is important in the genesis of nosocomial infections in the ICU, suggesting that local defects in host defence contribute to infection [9]. Furthermore, and while nosocomial infections are of major clinical importance, their harm, in terms of mortality, has not been well defined. Unadjusted studies show an association between the occurrence of ventilator-associated pneumonia and mortality. After adjustment for factors that independently influence the occurrence of ventilator-associated pneumonia and death, such as severity and progression of underlying illness, however, an independent effect on mortality has not been demonstrated in several large studies [10-12].

The contribution of a gene to bacterial virulence is defined by the molecular Koch's postulates [13]. It is not necessary to fulfil all postulates but a gene is more likely to contribute to virulence if it is present in pathogens but absent from closely related nonpathogenic organisms, if inactivation of the gene (via genetic engineering) results in loss of a virulent phenotype, and if replacement by an intact copy of the inactivated gene results in restoration of virulence [13]. Within the bacterial genome, virulence genes are often organised together in contiguous regions known as pathogenicity islands [14]. These packages often contain a set of genes, the products of which contribute to a specific virulence function, such as a type III secretion system (T3SS). Bacteria, unlike higher organisms, can transfer genetic material within and across species boundaries by horizontal transfer. Pathogenicity islands that contain similar genes and serve the same function have been identified in pathogens that have no recent common ancestor. This capacity for horizontal gene transfer is responsible for the wide and rapid spread of antibiotic resistance genes but has also served, over a longer evolutionary time period, to spread common mechanisms of virulence amongst diverse pathogens. Bacterial genes that contribute to virulence are often not expressed constitutively but rather are induced only following contact with or invasion of a host [15]. The expression of such genes in vivo is dependent on the pathogen having the capacity to sense its immediate environment sufficiently to identify contact with the host.

A repeating theme in bacterial virulence is that many, although not all, mechanisms of virulence are mediated by the subversion of host biological processes [16]. This involves specific (physical) interaction between the products of bacterial virulence genes and the host molecules that lead to alteration in host biological functions that serve the purposes of the pathogen, such as to survive and proliferate. A range of host cell functions have been shown to be subverted by bacterial pathogens, including a variety of signalling cascades ultimately resulting in reorganisation of the cytoskeletal apparatus during invasion of the host cell, inhibition of phagocytosis by host immune cells, and either promotion or inhibition of host cell apoptosis.

A system for classification of mechanisms of virulence is outlined in Table 1. The remainder of the present review describes selected mechanisms of virulence in greater depth. Those examples chosen for further discussion have been selected either because they illustrate important themes or principles or because they have particular relevance to infections that occur in the ICU. Many of the listed examples of mechanisms have, of necessity, been elucidated using models in which bacteria interact with host cells, often in cell culture, rather than with intact animals. The major purpose of this section is to describe the molecular basis of the host-pathogen interaction. The biological importance of these interactions has been established, for some mechanisms, using intact animals - although for some infections the absence of suitable models of infection precludes this.

\section{Adhesion}

Physical attachment of bacteria to host tissues, termed adhesion, is a critical component of almost all bacteria-host interactions. Adhesion can be divided into two broad categories: initial colonisation of the host surface via specific interactions with host receptors, and intimate association of 


\section{Table 1}

\section{Classification of bacterial virulence mechanisms}

1. Adhesion

- Loose adhesion

- Intimate adhesion

2. Invasion

- Transcellular (uptake across cell membranes using host cell uptake mechanisms, such as phagocytosis and microfold cell sampling or pathogen-directed endocytosis)

- Intercellular (traversal of an epithelial barrier between epithelial cells)

3. Intracellular survival mechanisms

- Within cytoplasm following escape from phagosome or endocytic vesicle

- Within an endocytic vesicle via avoidance of phagolysosome formation or autophagocytic pathway

- Prevention of host cell apoptosis

4. Extracellular survival mechanisms

- Antiphagocytic mechanisms (such as triggering of phagocyte apoptosis, subversion of lysosome fusion with the phagosome, resistance to oxygen free radicals)

- Serum resistance via preventing complement activation on the bacterial cell surface and inhibition of membrane attack complex insertion into the bacterial membrane

5. Nutrient acquisition

- Iron acquisition systems

6. Damage host cells and tissues

- Cytotoxins

- Enzymes that degrade extracellular matrix components

7. Motility

- Swimming (for example, flagella)

- Twitching motility (for example, type IV pili)

8. Biofilm formation

9. Regulation of virulence

- Sense environment and regulate transcription/activation of virulence genes

- Sense other bacteria (quorum sensing) and regulate transcription/activation of virulence genes

the bacteria with the host cell surface leading to invasion (see following sections).

The airway epithelium is the first point of contact for Neisseria meningitidis, Pseudomonas aeruginosa, Staphylococcus aureus and streptococci. These pathogens must overcome a variety of mechanical impediments to initiate contact with this epithelium. The airway epithelium consists of a variety of cell types, including squamous epithelial cells, ciliated and nonciliated columnar cells, goblet cells and microfold cells. The goblet cells secrete mucin, which forms a gel-like barrier covering the cell surface. The beating ciliated cells ensure constant movement of the mucin across this surface.

Initial contact of bacterial pathogens with the airway epithelium cells occurs via pili, long hair-like structures that protrude from the surface of the bacteria and terminate with a bacterial adhesin that binds to specific cell surface receptors. Certain types of pili such as the type IV pili of meningococci and $P$. aeurginosa are retracted into the cell once the receptor is engaged, thus dragging the bacterial cell into close contact with the surface of the host cell [17]. Although it has been recently discovered that $S$. aureus and streptococci express pili, the role in disease is not known. It is, however, clear that these pili are unable to undergo retraction and therefore are apparently permanently extended [18].

\section{Invasion}

Intact epithelial surfaces are a highly effective barrier to invasion by pathogens. A capacity to breach intact epithelial surfaces is an important characteristic for many specialised bacterial pathogens [16]. In contrast, some pathogens are 
dependent on local defects in the epithelial surface to achieve invasion, such as occurs with wound infections or peritonitis secondary to perforation of the intestinal tract.

Most pathogens that have the capacity to cross intact epithelial surfaces do so by passing through (transcellular), rather than between (intercellular), the cells of the epithelial surface. Transcellular uptake is either cell initiated - for example, by microfold cells that sample and internalise luminal contents as part of immune surveillance [19] - or pathogen directed - in which the pathogen subverts host mechanisms, leading to internalisation of the bacteria. A well-characterised process of pathogen-initiated transcellular uptake is utilised by Salmonella enterica. Following tight adherence of the bacteria to enterocytes, a T3SS is utilised to inoculate bacterial effector proteins into the host cell cytoplasm. These proteins, SopE and SopE2, function as GTPases leading to activation of host protein regulators of the actin cytoskeleton. Activation of these host proteins, CDC42 and Rac, leads to rearrangement of actin so that the cell membrane protrudes, surrounds, and then engulfs the adherent bacteria, delivering the bacteria across the cell membrane and into the cytoplasm [20]. Similar mechanisms are possessed by a wide variety of pathogens, including Yersinia sp., Shigella sp., Escherichia coli, and $P$. aeruginosa.

\section{Subversion of phagocytosis to access a protected intracellular niche}

Many important host defence mechanisms, such as complement and antibodies, act only within extracellular spaces. Some pathogens possess specialised mechanisms that allow them to exploit the protection conferred by the intracellular environment of the host cell. One such mechanism of accessing the intracellular environment is subversion of phagocytosis. Normal phagocytosis commences with the engulfment of the pathogen by neutrophils or macrophages that bind the bacteria. This results in the rearrangement of the actin cytoskeletal apparatus to produce pseudopodia that extend around and engulf the bacteria. An internalised membrane-bound vesicle containing the bacterium, termed a phagosome, is ingested and fuses with lysozomes, resulting in the formation of a phagolysozome. The lysozomes deliver low acidity, reactive oxygen moieties, proteolytic enzymes, and antibacterial peptides into the vesicle, leading to the destruction of the engulfed bacteria [21].

Some intracellular pathogens, such as Legionella pneumophila, Coxiella brunetii, and Brucella abortus, are capable of arresting the maturation of the phagolysosome [21]. This prevents delivery of the effector molecules of the lysosome, resulting in a membrane-bound compartment that supports bacterial survival and proliferation. Other intracellular pathogens, such as Shigella sp. and Listeria sp., have the capacity to disrupt the phagosome membrane, prior to its maturation, allowing the bacteria to escape into the cytosol where they survive and proliferate [21].
For many bacteria the precise mechanism by which normal phagocytosis is subverted is increasingly well understood. For example, following phagocytic uptake L. pneumophila injects multiple effector proteins, many of which are structurally similar to eukaryotic proteins, into the cytosol of the host cell via the Icm/Dot type IV secretion system. Although many interactions remain to be elucidated, the type IV secretion system effector proteins act to recruit host encoded small GTPases Rab1 and Sar1 to the Legionellacontaining vacuole, thus preventing phagosome maturation [22-24]. The recruitment of the GTPases to the Legionellacontaining vacuole results in the vacuole acquiring characteristics that are similar to the endoplasmic reticulum [24] to which lysozomes cannot fuse, thus creating a protected niche for the bacteria. The lifecycle of $L$. pneumophila can also involve existence within water-borne amoeba, with the same process of avoidance of phagosome maturation mediated by interaction between effector proteins and highly conserved eukaryotic proteins that regulate membrane trafficking occurring in this host [22].

\section{Prevention of phagocytic uptake}

Many pathogens lack a specialised apparatus to subvert phagosomal maturation and use avoidance of phagocytosis as a necessary strategy for virulence. Bacteria with mechanisms that subvert uptake by neutrophils and macrophages include Yersinia sp., $P$. aeruginosa, and enteropathogenic $E$. coli [25]. Yersinia sp. utilise a T3SS to directly inoculate effector proteins into the cytoplasm of host phagocytic cells. These effector proteins, including YopH, YopE, and YopT, interact directly with host encoded proteins that regulate actin polymerisation, thus preventing the cell surface membrane rearrangements that lead to phagocytic internalisation $[26,27]$. Similarly, the T3SS of $P$. aeruginosa inoculates ExoT and ExoS into the cytosol of host cells. Although these pseudomonal effector proteins are unrelated to the Yop factors, they activate some of the same host targets (the Rho GTPases RhoA, Rac-1, and Cdc42), resulting in the paralysis of engulfment by the phagocytic cell $[25,28]$.

\section{Regulation of host cell apoptosis}

Several bacterial pathogens possess mechanisms to subvert host cell apoptosis, usually leading to the apoptotic destruction of host inflammatory cells. Pathogens that interact with host cells and induce apoptosis include Salmonella sp., Shigella sp., Streptococcus pneumoniae and $P$. aeruginosa [29-31]. In contrast, Chlamydia sp. and Mycobacterium tuberculosis act to inhibit apoptosis following invasion, thus preserving cells that act as their intracellular niche [29]. The mechanisms utilised by Salmonella sp. and Shigella sp. to induce apoptosis of neutrophils have been elucidated in each pathogen and involve the T3SS effector proteins SipB and IpaB, respectively. These proteins act in the neutrophil cytosol, binding to and activating host caspase 1, the activation of which leads to host cell apoptosis. This process is likely to be important in abrogating the neutrophil-mediated killing of 
pathogens once they have penetrated the gut epithelial surface [32].

\section{Serum resistance}

The complement cascade is an essential arm of the innate immune system as well as an effector of the adaptive immune system. Over 20 proteins and protein fragments make up the complement system, including serum proteins, serosal proteins, and cell membrane receptors that are produced constitutively and circulate in the blood stream. The activation of this system by the classical and alternate pathways leads to the opsonisation of the pathogen with $\mathrm{C} 3 \mathrm{~b}$ and its cleavage fragment iC3b. Complement receptors on phagocytes bind $\mathrm{C} 3 \mathrm{~b}$ or $\mathrm{C} 4 \mathrm{~b}$ and $\mathrm{iC} 3 \mathrm{~b}$, resulting in phagocytosis of the pathogen in the presence or absence of antibodies. If complement activation continues from $\mathrm{C} 3 \mathrm{~b}$ to the formation of C5-convertases $\mathrm{C5a}$ and $\mathrm{C} 5 \mathrm{~b}$, these molecules act as chemoattractants that recruit inflammatory cells to sites of infection. Ultimately the pathway also results in the formation of the membrane attack complex (MAC) that inserts into the outer membrane of pathogen, forming pores that eventually lead to destruction of the bacterial cell. Host surfaces are protected from complement attack by host encoded inhibitors such as Factor $\mathrm{H}$ and $\mathrm{C} 4 \mathrm{~b}$-binding protein. Host Factor $\mathrm{H}$ binds cell surface polyanions such as terminal sialic acid on glycoproteins, and accelerates the decay of $\mathrm{C} 3 \mathrm{~b}$ into inactive iC3b. Similarly C4b-binding protein prevents the formation of new convertases by proteolytically degrading C4b [33].

Bacterial pathogens have adopted four main strategies to overcome the complement cascade: restricting the formation of $\mathrm{C} 3 \mathrm{~b}$ and $\mathrm{C} 4 \mathrm{~b}$ on the bacterial cell surface, the acquisition of Factor $\mathrm{H}$ and $\mathrm{C} 4 \mathrm{~b}$-binding protein to their own cell surfaces to downregulate activation of convertases on the bacterial cell surface, the inactivation of $\mathrm{C} 5 \mathrm{a}$ to prevent recruitment of inflammatory cells to the site of infection, and the inhibition of MAC insertion [33]. Resistance to insertion of the MAC is critically important to the serum resistance that is a characteristic of many pathogens. Gram-positive cell walls are intrinsically resistant to insertion of the MAC (Lambris). Among many Gram-negative organisms, the presence of smooth lipopolysaccharide results in resistance to the MAC. The rarity of bacteraemia caused by enteric Gram-negative organisms with rough lipopolysaccharide reflects the importance of this mechanism of serum resistance.

Furthermore, other Gram-negative serum-resistant pathogens, such as $N$. meningitidis and $\mathrm{K} 1$ strains of $E$. coli that cause neonatal meningitis, have serum resistance as a consequence of the protection conferred by sialic acid-containing capsules that prevent penetration of the MAC. In some instances, pathogens do not rely on one mechanism to become resistant to complement but use a collage of strategies. For example, N. meningitidis, in addition to its sialic acid capsule that restricts MAC insertion, possesses other mechanisms of serum resistance, including the major surface glycolipid lipopolysaccharide (lipo-oligosaccharide) that excludes C4b deposition, whilst surface proteins such as type IV pili and PorA attract C4b-binding protein, and the OMP GNA1870 binds Factor H [34]. Similarly, Group B $\beta$-haemolytic streptococci express a sialic acid capsule that restricts $\mathrm{C} 3 \mathrm{~b}$ deposition on the bacterial surface, an outer surface protein (Bac) acts as a filamentous Factor $\mathrm{H}$ binding protein, and $\mathrm{C} 5 \mathrm{a}$ is directly inactivated by the bacterial $\mathrm{C} 5 \mathrm{a}$ peptidase [35]. In these examples, it has been shown that some of these strategies play a more predominant role than others in the virulence of these organisms. For example, C5a peptidase is not expressed by all invasive Group B $\beta$-haemolytic streptococci although it is clear that inflammation in the host is reduced when it is not expressed by the pathogen [35].

\section{Quorum sensing}

Quorum sensing is an interbacterial signalling system that provides a link between the local density of bacteria and the regulation of gene expression. The sensing allows a population of bacteria to coordinate their gene expression in a manner that is dependent on the number of colocated bacteria. Quorum sensing is used by some pathogens, most notably $P$. aeruginosa, to coordinate the expression of virulence genes. This allows populations of bacteria to adopt virulent behaviour but only when a critical mass of bacteria is present [36].

The quorum sensing system of $P$. aeruginosa comprises two separate but interrelated systems, $r h /$ and las, both of which utilise (different) acyl homoserine lactones as signal transducers. The acyl homoserine lactones are secreted into the local environment with concentrations increasing in relation to bacterial numbers. Above a threshold intracellular concentration, the secreted acyl homoserine lactone molecules passively re-enter the cytosol of the bacteria, binding to and activating transcriptional regulators - which results in the expression of a range of genes that contribute to virulence [36]. Experimental inactivation of the $r h /$ and las systems results in marked attenuation of pseudomonal virulence in animal models of burns and pneumonia [36,37].

\section{Biofilm formation}

Biofilms are self-assembling, multicellular, communities of bacteria attached to a surface and enclosed within a selfsecreted exopolysaccharide matrix [38]. Bacteria that are capable of forming biofilms can switch between a free-living, or planktonic, form or existing within a biofilm. A mature biofilm is comprised of micro-colonies of bacteria within an exopolysaccharide matrix that is interspersed with water-filled channels that supply nutrients and remove wastes. The exopolysaccharide matrix is responsible for the sliminess of biofilms.

Biofilms are particularly resistant to many forms of physical and chemical insult, including antibiotics. Important patho- 
gens that have a propensity for biofilm formation include $S$. aureus, coagulase-negative Staphylococci, and $P$. aeruginosa [38]. In the ICU, biofilms are particularly important for infection and colonisation of devices such as intravascular catheters, urinary catheters, endotracheal tubes, and prosthetic heart valves.

Bacteria within a biofilm are highly tolerant to antibiotics, even when planktonic derivatives of a biofilm demonstrate high degrees of in vitro sensitivity to the same antibiotic. The origin of biofilm tolerance to antibiotics is multifactorial but includes reduced penetration of antibiotics into the biofilm matrix and the presence of metabolically inactive dormant cells [39]. The functional resistance of biofilm-associated infections to antibiotics explains the importance of removal of infected devices to successful clearance of infection.

\section{Bacterial virulence - evolutionary origin}

The mechanisms of bacterial virulence that have been described represent only a small selection among many different strategies. Nevertheless, those chosen are representative and serve to illustrate that bacterial virulence frequently involves specific interactions, at a molecular level, between bacterial encoded structures with host molecules that leads directly to the subversion of host cell functions to provide a survival advantage for the bacteria. These mechanisms have presumably developed over hundreds of millions of years of coexistence of bacterial pathogens and hosts [40]. That mechanisms of such sophistication have developed reflects the power of vertical evolutionary change in organisms with short generation times coupled to the spread of genes that confer advantage by horizontal genetic exchange.

\section{Clinical implications}

The elucidation of the subversion of host mechanisms to promote bacterial virulence has been of major scientific interest, reflecting the elegance and sophistication of these mechanisms. The pathogens that have been most intensively studied are those that are capable of virulence irrespective of the presence of defects in local or systemic host immunity.

Many infections that necessitate admission to the ICU occur in the context of a previously healthy host and involve no obvious defect in local or systemic immunity. Examples of these types of infection include overwhelming meningococcal sepsis and some patients with community-acquired pneumonia, urosepsis, and skin and soft tissue infections. Many patients with infection that leads to ICU admission, however, possess underlying defects that predispose them to infection, including systemic factors such as pharmacological immunosuppression, malignancy, and diabetes or local defects such as obstructed or perforated viscus, invasive devices, or surgical wounds. The majority of nosocomial infections acquired in the ICU are also heavily influenced by local and systemic defects in host immunity, particularly the presence of invasive devices (van der Kooi). While factors such as capsulation and serum resistance are likely to be critical in the establishment of nosocomial infection, the importance of more elaborate mechanisms of bacterial virulence to infections that occur in this context is less certain. In general, there has been much less investigation of mechanisms of virulence in pathogens of clinical relevance to intensive care, at least in part because of the paucity of characterised and validated animal models of nosocomial infections. There is good evidence for the probable importance of mechanisms such as biofilm formation, quorum sensing, and serum resistance in many infections of relevance to ICU patients.

The potential value of mechanisms of virulence as a therapeutic or prophylactic target is speculative. There is clear proof-of-principle that therapeutic targeting of the regulation of a virulence mechanism can prevent disease by a pathogen [41]. The bacteria responsible for most serious infections, however, are killed rapidly by antibiotics and it is uncertain whether a drug that targeted virulence would have any value as an alternative or supplement to antibiotics. Furthermore, since mechanisms of virulence are often restricted to a specific pathogen and there can be redundancy among mechanisms of virulence in many pathogens, this type of targeted intervention may have limited clinical utility.

At the present time there is little enthusiasm in industry for the development of small-molecule drugs that target virulence mechanisms. This is despite two theoretical attractions to targeting virulence. Firstly, at least conceptually, there is a potentially attractive role for drugs that target virulence in the prevention of ICU-related nosocomial infections. Antibiotics, which kill commensal as well as pathogenic bacteria indiscriminately, result in undesired effects such as selection of antibiotic-resistant organisms and altered mucosal function. In contrast, an agent that acted to prevent virulence of a specific pathogen would leave the commensal flora intact. Although such agents will be highly specific, effective pharmacoprophylaxis for important nosocomial pathogens, such as $P$. aeruginosa or $S$. aureus - for example, by blockade of quorum sensing - might have clinical utility [42]. The importance of preventing nosocomial infections, by any means, is only likely to increase in association with worsening antibiotic resistance. Secondly, some forms of infection that are clinically important in ICU populations are not amenable to treatment solely with antibiotics. Research of biofilmrelated infections is particularly active and raises the prospect of control of device-related infection without the need to remove the device [43].

\section{Conclusion}

The mechanisms by which bacterial pathogens interact with and subvert host defence mechanisms are being rapidly defined for a wide range of pathogens. While these mechanisms are likely to be relevant to infections that necessitate ICU admission in patients with normal host defences, however, the relevance of these mechanisms to 


\section{This article is part of a review series on Infection, edited by Steven Opal.}
Other articles in the series can be found online at http://ccforum.com/articles/ theme-series.asp?series $=\mathrm{CC} \_$Infection

infections that require local or systemic defects in host defences remains to be established. While there are theoretical rationales for the development of agents that target virulence, particularly for nosocomial pathogens, the restriction of specific virulence mechanisms to a narrow range of pathogens may limit utility.

\section{Competing interests}

The authors declare that they have no competing interests.

\section{References}

1. Kumar A, Roberts D, Wood KE, Light B, Parrillo JE, Sharma S, Suppes R, Feinstein D, Zanotti S, Taiberg L, Gurka D, Cheang M: Duration of hypotension before initiation of effective antimicrobial therapy is the critical determinant of survival in human septic shock. Crit Care Med 2006, 34:1589-1596.

2. Finfer S, Bellomo R, Lipman J, French C, Dobb G, Myburgh J: Adult-population incidence of severe sepsis in Australian and New Zealand intensive care units. Intensive Care Med 2004, 30:589-596.

3. Vandenbroucke-Grauls CM, Vandenbroucke JP: Effect of selective decontamination of the digestive tract on respiratory tract infections and mortality in the intensive care unit. Lancet 1991, 338:859-862.

4. Lim SM, Webb SA: Nosocomial bacterial infections in intensive care units. I: organisms and mechanisms of antibiotic resistance. Anaesthesia 2005, 60:887-902.

5. Overbye KM, Barrett JF: Antibiotics: where did we go wrong? Drug Discov Today 2005, 10:45-52.

6. Falkow S: What is a pathogen? Am Soc Microbiol News 1991, 63:356-359.

7. Hoffmann JA: The immune response of Drosophila. Nature 2003, 426:33-38.

8. Falkow S: The evolution of pathogenicity. In Escherichia coli and Salmonella cellular and molecular biology. Edited by Neidhardt FC. Washington, DC: ASM Press; 1996.

9. van der Kooi TI, de Boer AS, Mannien J, Wille JC, Beaumont MT, Mooi BW, van den Hof S: Incidence and risk factors of deviceassociated infections and associated mortality at the intensive care in the Dutch surveillance system. Intensive Care Med 2007, 33:271-278.

10. Wolkewitz M, Vonberg RP, Grundmann H, Beyersmann J, Gastmeier P, Barwolff S, Geffers C, Behnke M, Ruden H, Schumacher M: Risk factors for the development of nosocomial pneumonia and mortality on intensive care units: application of competing risks models. Crit Care 2008, 12:R44.

11. Papazian L, Bregeon F, Thirion X, Gregoire R, Saux P, Denis JP, Perin G, Charrel J, Dumon JF, Affray JP, Gouin F: Effect of ventilator-associated pneumonia on mortality and morbidity. $\mathrm{Am} J$ Respir Crit Care Med 1996, 154:91-97.

12. Heyland DK, Cook DJ, Griffith L, Keenan SP, Brun-Buisson C: The attributable morbidity and mortality of ventilator-associated pneumonia in the critically ill patient. The Canadian Critical Trials Group. Am J Respir Crit Care Med 1999, 159: 1249-1256.

13. Falkow S: Molecular Koch's postulates applied to bacterial pathogenicity - a personal recollection 15 years later. Nat Rev Microbio/ 2004, 2:67-72.

14. Hacker J, Blum-Oehler G, Muhldorfer I, Tschape H: Pathogenicity islands of virulent bacteria: structure, function and impact on microbial evolution. Mol Microbiol 1997, 23:1089-1097.

15. Mahan MJ, Heithoff DM, Sinsheimer RL, Low DA: Assesment of bacterial pathogenesis by analysis of gene expression in the host. Annu Rev Genet 2000, 34:139-164.

16. Hornef MW, Wick MJ, Rhen M, Normark S: Bacterial strategies for overcoming host innate and adaptive immune responses. Nat Immunol 2002, 3:1033-1040.

17. Shi W, Sun H: Type IV pilus-dependent motility and its possible role in bacterial pathogenesis. Infect Immun 2002, 70:1-4.

18. Scott JR, Zahner D: Pili with strong attachments: Gram-positive bacteria do it differently. Mol Microbiol 2006, 62:320-330.

19. Sansonetti PJ, Phalipon A: M cells as ports of entry for enteroinvasive pathogens: mechanisms of interaction, consequences for the disease process. Semin Immunol 1999, 11: 193-203.

20. Friebel A, Ilchmann H, Aepfelbacher M, Ehrbar K, Machleidt W, Hardt WD: SopE and SopE2 from Salmonella typhimurium activate different sets of RhoGTPases of the host cell. $J$ Biol Chem 2001, 276:34035-34040.

21. Meresse S, Steele-Mortimer O, Moreno E, Desjardins M, Finlay B, Gorvel JP: Controlling the maturation of pathogen-containing vacuoles: a matter of life and death. Nat Cell Biol 1999, 1: E183-E188.

22. Bruggemann $\mathrm{H}$, Cazalet $\mathrm{C}$, Buchrieser $\mathrm{C}$ : Adaptation of Legionella pneumophila to the host environment: role of protein secretion, effectors and eukaryotic-like proteins. Curr Opin Microbiol 2006, 9:86-94.

23. Nagai $H$, Kagan JC, Zhu $X$, Kahn RA, Roy CR: A bacterial guanine nucleotide exchange factor activates ARF on Legionella phagosomes. Science 2002, 295:679-682.

24. Kagan JC, Stein MP, Pypaert M, Roy CR: Legionella subvert the functions of Rab1 and Sec22b to create a replicative organelle. J Exp Med 2004, 199:1201-1211.

25. Rosenberger CM, Finlay BB: Phagocyte sabotage: disruption of macrophage signalling by bacterial pathogens. Nat Rev Mol Cell Biol 2003, 4:385-396.

26. Persson C, Carballeira N, Wolf-Watz H, Fallman M: The PTPase YopH inhibits uptake of Yersinia, tyrosine phosphorylation of p130Cas and FAK, and the associated accumulation of these proteins in peripheral focal adhesions. Embo J 1997, 16:23072318.

27. Knodler LA, Celli J, Finlay BB: Pathogenic trickery: deception of host cell processes. Nat Rev Mol Cell Biol 2001, 2:578-588.

28. Goehring UM, Schmidt G, Pederson KJ, Aktories K, Barbieri JT: The N-terminal domain of Pseudomonas aeruginosa exoenzyme $\mathrm{S}$ is a GTPase-activating protein for Rho GTPases. J Biol Chem 1999, 274:36369-36372.

29. Merrell DS, Falkow S: Frontal and stealth attack strategies in microbial pathogenesis. Nature 2004, 430:250-256.

30. Lau GW, Hassett DJ, Britigan BE: Modulation of lung epithelial functions by Pseudomonas aeruginosa. Trends Microbiol 2005, 13:389-397.

31. Marriott HM, Dockrell DH: Streptococcus pneumoniae: the role of apoptosis in host defense and pathogenesis. Int J Biochem Cell Biol 2006, 38:1848-1854.

32. Hilbi H, Moss JE, Hersh D, Chen Y, Arondel J, Banerjee S, Flavell RA, Yuan J, Sansonetti PJ, Zychlinsky A: Shigella-induced apoptosis is dependent on caspase-1 which binds to IpaB. J Biol Chem 1998, 273:32895-32900.

33. Kraiczy P, Wurzner R: Complement escape of human pathogenic bacteria by acquisition of complement regulators. Mol Immunol 2006, 43:31-44.

34. Schneider MC, Exley RM, Ram S, Sim RB, Tang CM: Interactions between Neisseria meningitidis and the complement system. Trends Microbiol 2007, 15:233-240.

35. Jarva H, Jokiranta TS, Wurzner R, Meri S: Complement resistance mechanisms of streptococci. Mol Immunol 2003, 40:95-107.

36. Smith RS, Iglewski $\mathrm{BH}: \boldsymbol{P}$. aeruginosa quorum-sensing systems and virulence. Curr Opin Microbiol 2003, 6:56-60.

37. Rumbaugh KP, Griswold JA, Iglewski BH, Hamood AN: Contribution of quorum sensing to the virulence of Pseudomonas aeruginosa in burn wound infections. Infect Immun 1999, 67: $5854-5862$.

38. Costerton W, Veeh R, Shirtliff M, Pasmore M, Post C, Ehrlich G: The application of biofilm science to the study and control of chronic bacterial infections. J Clin Invest 2003, 112:1466-1477. 
39. Drenkard E: Antimicrobial resistance of Pseudomonas aeruginosa biofilms. Microbes Infect 2003, 5:1213-1219.

40. Strauss E: Microbiology. Simple hosts may help reveal how bacteria infect cells. Science 2000, 290:2245-2247.

41. Hung DT, Shakhnovich EA, Pierson E, Mekalanos JJ: Small-molecule inhibitor of Vibrio cholerae virulence and intestinal colonization. Science 2005, 310:670-674.

42. Otto M: Quorum-sensing control in Staphylococci - a target for antimicrobial drug therapy? FEMS Microbiol Lett 2004, 241:135-141.

43. Valle J, Da Re S, Henry N, Fontaine T, Balestrino D, LatourLambert P, Ghigo JM: Broad-spectrum biofilm inhibition by a secreted bacterial polysaccharide. Proc Natl Acad Sci U S A 2006, 103:12558-12563. 